1. C. B. Boyer, History of Analytic Geometry, Scripta Mathematica, New York, 1956.

2. J. L. Coolidge, The origin of analytic geometry, Osiris 1 (1936) 231-250, also available at www. jstor. org.

3. M. Ghomi and B. Solomon, Skew loops and quadric surfaces, Comment. Math. Helv. 4 (2002) 767-782.

4. J.-P. Sha and B. Solomon, No skew branes on non-degenerate hyperquadrics, Math. Zeit. 257 (2002) 225229.

5. S. Tabachnikov, On skew loops, skew branes, and quadratic hypersurfaces, Moscow Math. J. 3 (2003) 681-690.

Math Department, Indiana University, Bloomington, IN 47405

solomon@indiana.edu

\title{
On Furstenberg's Proof of the Infinitude of Primes
}

\section{Idris D. Mercer}

Theorem. There are infinitely many primes.

Euclid's proof of this theorem is a classic piece of mathematics. And although one proof is enough to establish the truth of the theorem, many generations of mathematicians have amused themselves by coming up with alternative proofs. See, for example, [2], or Chapter 1 of either [1] or [4].

There is a particularly striking proof, due to Furstenberg in 1955 [3], that uses, of all things, topological language! In this note, we give a variant of Furstenberg's proof that avoids topological language and thus, in the opinion of the current author, better exhibits the "real reason" that Furstenberg's approach works.

Definition. If $m$ and $r$ are integers with $m \geq 1$, we let $r+m \mathbf{Z}$ denote the set of integers congruent to $r \bmod m$, so for example,

$$
2+7 \mathbf{Z}=9+7 \mathbf{Z}=-5+7 \mathbf{Z}=\{\ldots,-12,-5,2,9,16, \ldots\} .
$$

We call any such set an arithmetic progression, or AP for short.

Notation. For $m \geq 2$, the set of integers not divisible by $m$ is

$$
(1+m \mathbf{Z}) \cup \cdots \cup((m-1)+m \mathbf{Z}),
$$

which we abbreviate by $\mathrm{NM}(m)$ (which stands for "non-multiples" of $m$ ).

Claim 1. A finite intersection of APs is either empty or infinite.

Proof. If $x$ belongs to $r_{i}+m_{i} \mathbf{Z}$ for $1 \leq i \leq k$, then so does $x+y$ where $y$ is any common multiple of the $m_{i}$. 
Claim 2. If $\mathcal{S}$ is any collection of sets, then a finite intersection of finite unions of sets in $\mathcal{S}$ is also a finite union of finite intersections of sets in $\mathcal{S}$.

Proof. This just states the fact that intersection distributes over union. For example, we have

$$
\begin{aligned}
(R \cup S \cup T) \cap(U \cup V) \cap(W \cup X)= & (R \cap U \cap W) \cup(R \cap U \cap X) \\
& \cup(R \cap V \cap W) \cup(R \cap V \cap X) \\
& \cup(S \cap U \cap W) \cup(S \cap U \cap X) \\
& \cup(S \cap V \cap W) \cup(S \cap V \cap X) \\
& \cup(T \cap U \cap W) \cup(T \cap U \cap X) \\
& \cup(T \cap V \cap W) \cup(T \cap V \cap X) .
\end{aligned}
$$

Proof of Theorem. If $p_{1}, \ldots, p_{k}$ were all the primes, we would have

$$
\{-1,+1\}=\mathrm{NM}\left(p_{1}\right) \cap \mathrm{NM}\left(p_{2}\right) \cap \cdots \cap \mathrm{NM}\left(p_{k}\right),
$$

which is a finite intersection of finite unions of APs and hence, by Claim 2, a finite union of finite intersections of APs, which by Claim 1 must be either empty or infinite. This is a contradiction.

\section{REFERENCES}

1. M. Aigner and G. M. Ziegler, Proofs from The Book, Springer-Verlag, New York, 1998.

2. C. K. Caldwell, Proofs that there are infinitely many primes (2007), available at http://primes.utm. edu/notes/proofs/infinite.

3. H. Furstenberg, On the infinitude of primes, this Monthly 62 (1955) 353.

4. P. Ribenboim, The New Book of Prime Number Records, 3rd ed., Springer-Verlag, New York, 1996.

\section{Mean, Meaner, and the Meanest Mean Value Theorem}

\section{J. J. Koliha}

... the real nature of the Mean Value Theorem is exhibited by writing it as an inequality.

—J. Dieudonné

1. INTRODUCTION. The Mean Value Theorem of the elementary calculus keeps attracting the attention of mathematicians who ponder how to make its proof simple and elegant [1], how to generalize it [10], how to use it in proofs of other theorems [8], and, perversely, how to avoid it [2]. This MONTHLY has carried dozens of articles in which the Mean Value Theorem was the hero or the villain (for instance [5]). 